Historic, Archive Document

Do not assume content reflects current scientific knowledge, policies, or practices. 



\section{Price List to the Trade}
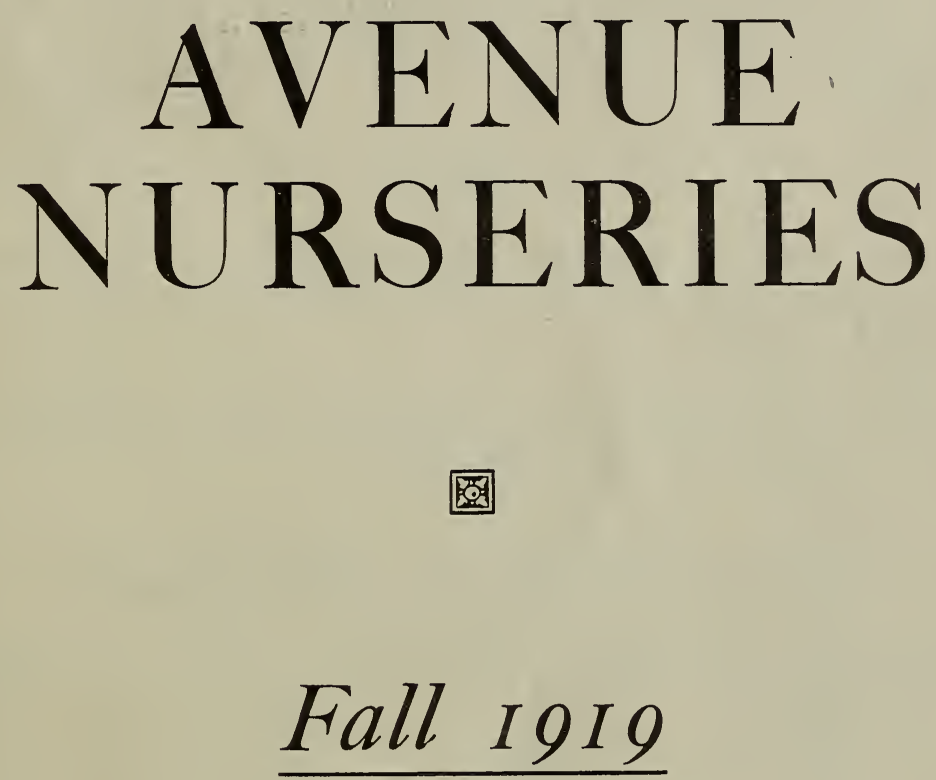

W. B. COLE, Proprietor PAINESVILLE, OHIO

Office and grounds one and one-half miles west of Painesville, on Mentor Avenue, and 28 miles east of Cleveland. C. P. छ E. cars every hour from Cleveland and Painesville. Stop 79 is in front of our office and grounds. Bell Telephone No. 97-R. 


\section{W. B. COLE, NURSERYMAN, PAINESVILLE, OHIO}

\section{RESPONSIBILITY}

We endeavor to send our patrons the grade and quality of stock ordered. We do not guarantee our products or assume any responsibility after they leave our nursery, as conditions always exist over which we have no control. It is understood, in case of error on our part, that we shall not be held responsible for a greater amount than the price paid.

\section{TERMS}

Two per cent. off for cash before shipment. To parties having satisfactory credit arrangement or account with us, one per cent. ten days, thirty days net.

No claims for rejections or deficiencies will be entertained unless made within ten days after arrival of goods.

\section{PRICES FOR BOXING AND PACKING}

Boxes, $32 \times 32$ inches, 10 feet long

\begin{tabular}{|c|c|c|c|c|c|}
\hline $32 \times 32$ & “ & 8 & " & “" & 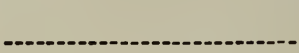 \\
\hline $28 \times 28$ & “ & 10 & " & 6 & 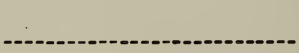 \\
\hline $28 \times 28$ & “6 & 8 & 66 & “ & \\
\hline $24 \times 24$ & “6 & 10 & “ & “ & \\
\hline $4 \times 24$ & “ & 8 & “ & “6 & \\
\hline $0 \times 20$ & “6 & 10 & 66 & “6 & \\
\hline $20 \times 20$ & “ & 8 & “6 & “6 & \\
\hline $6 \times 16$ & “6 & 10 & “6 & “ & \\
\hline $6 \times 1$ & “ & 8 & “6 & “6 & $\ldots$ \\
\hline
\end{tabular}

For $12 \mathrm{ft}$, boxes, add $\$ 1.00$ to price of $10 \mathrm{ft}$. boxes.

Bales 50 cts. to $\$ 3.00$, according to size.

35 cts. to 75 cts. each charged for paper lining boxes.

Boxes for small fruit plants, 25 cts. to $\$ 1.50$ each, according to size.

Rye Straw for baling, per bundle. $\$ .15$

Burlap, per $1 \mathrm{~b}$. .15

Paper, per 16.

Labels, Wired, per 1,000 2.00

List of varieties and prices subject to change to conform to our stock and condition of trade at the time the order is given. 
W. B. COLE, NURSERYMAN, PAINESVILLE, OHIO

\section{Fruit Department}

\section{APPLES, Standard}

5 to 6 feet, $\frac{11}{16}$ inch

Per 100 Per 1000

4 to 5 feet, $5 / 8$ inch.

$\$ 35.00 \$ 300.00$

$3 \frac{1}{2}$ to 4 feet, $1 / 2$ inch

$30.00 \quad 250.00$

$20.00 \quad 160.00$

\section{APPLES, Dwarf}

3 to 4 feet, $5 / 8$ inch

Per 10 Per 100 Per 1000

3 to 4 feet, $1 / 2$ inch

$\$ 35.00$

$\$ 300.00$

$\begin{array}{lll}3.50 & 30.00 \quad 250.00\end{array}$

All varieties can be supplied in standards; varieties starred (*) in dwarfs.

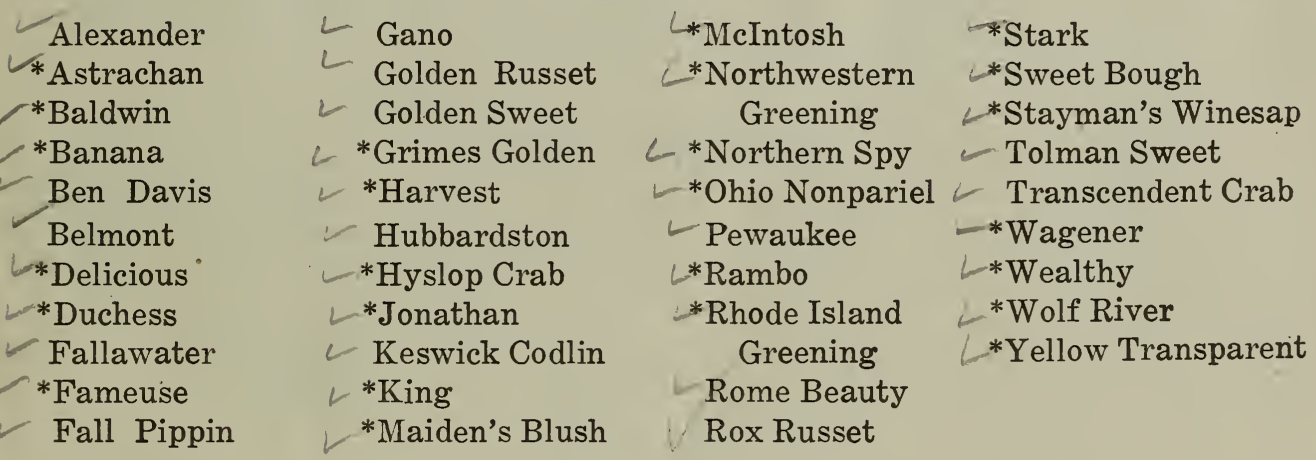

PEARS, Standard

5 to 6 feet, $\frac{1 x}{16}$ inch

Per 100 Per 1000

$\$ 35.00 \quad \$ 300.00$

4 to 5 feet, $5 / 8$ inch

$30.00 \quad 250.00$

4 to 5 feet, $1 / 2$ inch

25.00

200.00

$\checkmark$ Worden-Seckel and Beurre Bosc $5 \mathrm{cts}$. each extra.

\section{PEARS, Dwarf}

$31 / 2$ to 4 ftet, $5 / 8$ inch

Per 100 Per 1000

3 to 4 feet, $1 / 2$ inch

$\$ 25.00$

$\$ 220.00$

$20.00 \quad 180.00$

All varieties can be supplied in standards; varieties starred (*) in dwarfs.

$\checkmark *$ Anjou

*Bartlett

* Clarigeau

Beurre Bosc

*Clapp's Favorite
$*$ Duchess

Flemish Beauty

Howell

*Kieffer

Koonce
*Lawrence

$\checkmark *$ Louise Bonne

*Mary

*Rosney

*Seckel
Sheldon

*Vermont Beauty

*Wilder

*Worden Seckel 


W. B. COLE, NURSERYMAN, PAINESVILIE, OHIO

\section{PLUMS}

5 to 6 feet, $\frac{11}{16}$ inch

Per 100

$\$ 40.00$

Per 1000

4 to 5 feet, $5 / 8$ inch

35.00

$\$ 370.00$

3 to 4 feet, $1 / 2$ inch

30.00

320.00

250.00

Abundence

Arch Duke

Bradshaw

Burbank

Climax

Coes Golden

Diamond

$\begin{array}{ll}\text { Fellenburg (York } & \text { Lombard } \\ \text { State Prune) } & \text { Monarch } \\ \text { French Damson } & \text { Moore's Arctic } \\ \text { German Prune } & \text { Niagara } \\ \text { Grand Duke } & \text { October Purple } \\ \text { Geuii } & \text { Pond } \\ \text { Imperial Gage } & \text { Red June }\end{array}$

CHERRIES, Sweet
Per 100

$\$ 40.00$

35.00

30.00
Per 1000

$\$ 370.00$

300.00

250.00

4 to 5 feet, $1 / 2$ inch

Per 100

$\$ 40.00$

35.00

30.00
Per 1000

$\$ 370.00$

300.00

250.00

3 to 4 feet $1 / 2$ inch
Dyehouse
May Duke
Montmorency
Philippe
Richmond
Hortense
Morello
Wragg

\section{APRICOTS}

4 to 6 feet, $\frac{11}{16}$ inch

Per 10 Per 100

4 to 5 feet, $5 / 8$ inch

$\$ 4.00 \$ 35.00$

$3.50 \quad 30.00$

\section{PEACHES}

4 to 6 feet, $\frac{9}{16}$ inch

Per 100 Per 1000

$\$ 30.00$

25.00

$\$ 270.00$

220.00

3 to 4 feet, $\frac{7}{16}$ inch

20.00

170.00

2 to 3 feet, whips

$15.00 \quad 120.00$

Rochester, 5 cts. each extra.

Admiral Dewey

Alexander

Banner

Beer's Smock

Belle of Georgia

Captain Ede

Carmen

Chair's Choice

Champion

Crawford's Early

Crawford's Late

Crosby
Early Rivers

Early Elberta

Elberta

Fitzgerald

Foster

Gold Drop

Greensboro

Hale

Heath's Cling

Ingle's Mammoth

Kalamazoo

Lemon Cling
Lemon Free

Mayflower

Mathew's Beauty

Mountain Rose

New Prolific

Niagara

Old Mixon

Ray

Reeve's Favorite

Rochester

$\checkmark$ Salway

Scott's Nonpariel
Slappy

Smock Free

Stearns

Stump-the-World

Triumph

Wheeler's Late

Wilma

Yellow St. John

William's Cling 


\section{W. B. COLE, NURSERYMAN, PAINESVILIE, OHIO}

\section{QUINCES}

4 to 5 feet, $5 / 8$ inch

Per 10 Per 100

3 to 4 feet, $1 / 2$ inch

$\$ 4.00 \$ 35.00$

$3.00 \quad 25.00$

Borrgeat

$\checkmark$ Champion

Meeches

Corange

\section{MULBERRIES}

New American, 5 to 6 feet.

Per 10

8 to 10 feet

$\$ 6.00$

10.00

Russian, 4 to 6 feet

3.00

6 to 8 feet

5.00

\section{NUTS}

Butternut, 4 to 6 feet

6 to 8 feet

Per 10

$\$ 4.00$

6.00

8.00

3.00

Chestnut, American Sweet, 3 to 4 feet.

4.50

Paragon, 2 to 3 feet

6.00

3 to 4 feet

7.50

4 to 6 feet

10.00

5.00

Hickory, Shellbark, $1 \frac{1 / 2}{2}$ to 2 feet.

7.50

Pecans, 2 to 3 feet.

4.00

4 to 6 feet.

6 to 8 feet.

6.00

8 to 10 feet

8.00

Japan, 4 to 6 feet.

Per 100

$\$ 35.00$

50.00

70.00

25.00

40.00

40.00

35.00

50.00

70.00

\section{GOOSEBERRIES}

Downing, 1 year.

Per 10 Per 100

$\$ 12.00$

14.00

2 years

Houghton, 1 year.

7.00

9.00

Red Jacket, 2 years

$\$ 2.00 \quad 18.00$

Per 1000

$\$ 100.00$

120.00

\section{CURRANTS}

Black Champion, 1 year.

Cherry, 1 year.

2 years

12.00

Fays Prolific, 1 year..........

10.00

2 years

12.00

London Market, 1 year.

10.00

2 years

12.00

Perfection, 1 year

Red Cross, 1 year....................................................................................... 10.00

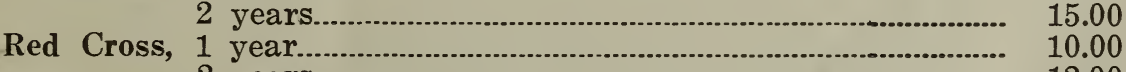

2 years.

White Grape, 1 year. 


\section{\begin{tabular}{lllll}
\hline \hline W. B. COLE, NURSERYMAN, PAINESVILLE, & OHIO \\
\hline \hline
\end{tabular}}

\section{GRAPE VINES}

Agawam, 1 year

Per 100 Per 1000

2 years

$\$ 12.00$

15.00

Brighton, 1 year

15.00

2 years

20.00

Campbell's Early, 1 year.

Catawba, 1 year.

10.00

2 years.

12.00

Concord, 1 year.

9.00

12.00

80.00

2 years

110.00

Delaware, 1 year.

15.00

2 years

20.00

Diamond, 1 year.

12.00

2 years

15.00

Moore's Early, 1 year.

15.00

2 years

18.00

Niagara, 1 year

12.00

2 years

15.00

Salem, 1 year.

12.00

2 years

15.00

Worden, 1 year.

12.00

2 years

15.00

\section{RASPBERRIES, Red}

Cuthbert

Per 100 Per 1000

King

$\$ 2.50 \$ 20.00$

Marlboro

2.50

St. Regis

2.50

3.00 


W. B. COLE, NURSERYMAN, PAINESVILLE, OHIO

\section{RASPBERRIES, Black}

Cumberland

Per 100 Per 1000

Gregg

$\$ 2.50$

$\$ 20.00$

Plum Farmer

20.00

$\begin{array}{ll}2.50 & 20.00 \\ 2.50 & 20.00\end{array}$

\section{RASPBERRIES, Root Cutting Plants}

Blowers

Per 100 Per 1000

Early Harvest

$\$ 3.50$

Early King

3.00

Eldorado

3.00

3.50

Lucretia

3.00

Mersereau

3.00

3.00

Ohmer

3.00

Taylor

3.00

3.00

25.00

Watt

25.00

25.00

25.00

25.00

25.00

\section{ASPARAGUS}

Palmetto, 2 years

Per 100

Per 1000

$\$ 1.00 \$ 8.00$

\section{RHUBARB}

Linneus and Queen, 2 years.

Per 100 Per 1000

$\$ 5.00 \$ 40.00$

\section{STRAWBERRIES}

(Delivery after October 1st.)

Leading Sorts

Per 100 Per 1000

$\$ \quad .75 \$ 6.00$

Pot-Grown plants in varieties starred $\left(^{*}\right)$ after September 1st 5.00
Brandywine
Gibson
*Bubach
*Glen Mary
Excelsior
Haverland
Gandy
Jessie
*Marshall
Parson's Beauty
Ryckman
*Sample

\section{Senator Dunlap}
Uncle Jim
William Belt

\section{STRAWBERRIES, Everbearing}

Progressive and Superb

Pot-Grown plants after September 1st. 
W. B. COLE, NURSERYMAN, PAINESVILLE, OHIO

ACER-Saccharum. Sugar or Rock Maple-

6 to 8 feet

8 to 19 feet.

Per 10

$\$ 5.50$

$11 / 4$ inch

7.50

$11 / 2$ inch

10.00

12.50

$13 / 4$ inch

2 inches

15.00

$21 / 2$ inches

3 inches

$31 / 2$ inches

4 inches

20.00

30.00

40.00

50.00

60.00

75.00

AESCULUS-Hippocastanum. European Horse Chestnut-

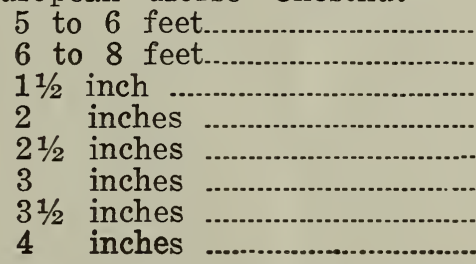

7.50

70.00

10.00

15.00

95.00

20.00

140.00

25.00

30.00

190.00

40.00

50.00

Hippocastanum Rubicunda. Red flowering Horse Chestnut. 8 to 10 feet.

25.00

ARALIA-Spinosa.

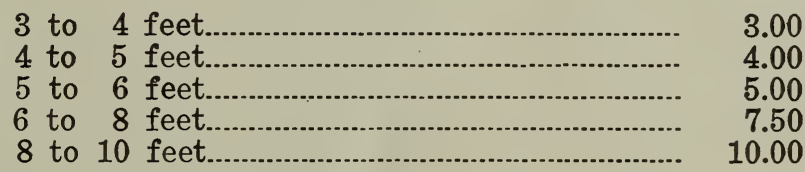

BETULA-Alba. European White Birch-

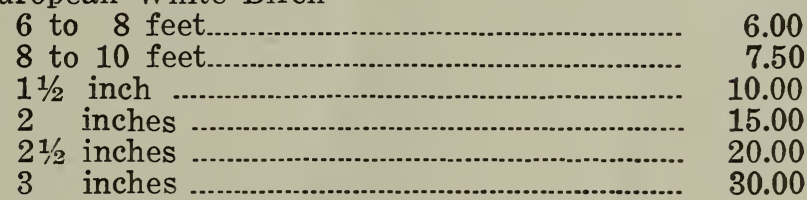

Alba Laciniata. Cut-leaved Weeping White Birch-

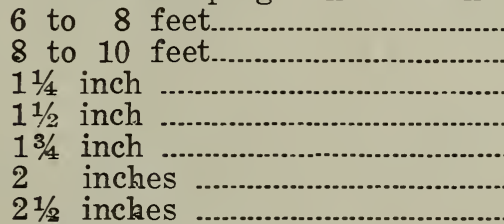

7.50

10.00

12.50

15.00

20.00

25.00

225.00

30.00

CATALPA-Bungei. 2 year heads,

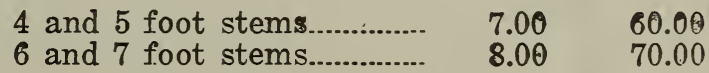

Speciosa.

$\begin{array}{lrr}4 \text { and } 5 \text { foot stems.............. } & 7.00 & 60.00 \\ 6 \text { and } 7 \text { foot stems............ } & 8.00 & 70.00\end{array}$

6 to 8 feet

$3.50 \quad 30.00$

8 to 10 feet.

$5.50 \quad 50.00$

$11 / 2$ inch

7.50

70.00

2 inches

12.50

115.00

$21 / 2$ inches

$16.00 \quad 150.00$

CERASUS-Japonica Rosea Pendula. Japan Weeping Cherry

20.00

FAGUS-Sylvatica Purpurea. Purple-leaved Beech-

3 to 4 feet

4 to 5 feet

5 to 6 feet

6 to 8 feet

20.00 


\section{W. B. COLE, NURSERYMAN, PAINESVILIE, OHIO}

FAGUS-Sylvatica Riversii. Blood-leaved Beech-

3 to 4 feet.

4 to 5 feet.

5 to 6 feet

6 to 8 feet.

Per 10 Per 100

$\$ 12.50$

15.00

20.00

25.00

FRAXINUS-Viridis Lanceolata. Green Ash-

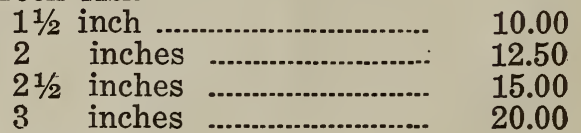

Americana. American Ash.

$$
\begin{array}{lr}
8 \text { to } 10 \text { feet............. } & 7.50 \\
11 / 2 \text { inch ................... } & 10.00
\end{array}
$$

LARIX-Europea. European Larch-

6 to 8 feet.

10.00

8 to 10 feet.

12.50

$1 \frac{1 / 2}{2}$ inch

15.00

Leptolepis. Japanese Larch-

$$
\begin{aligned}
& 6 \text { to } 8 \text { feet. } \\
& 8 \text { to } 10 \text { feet. } \\
& 11 / 2 \text { inch }
\end{aligned}
$$

10.00

12.50

15.00

LIQUIDAMBER_Styraciflua. Sweet Gum-

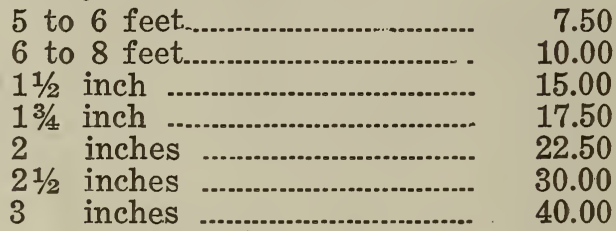

LIRODENDRON-Tulipifera. Tulip Tree-

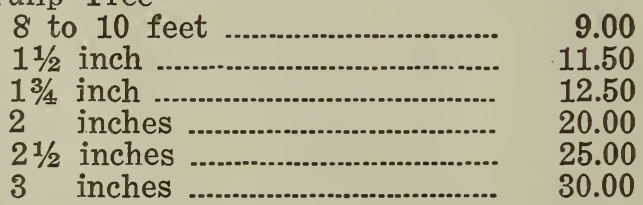

MAGNOILA-Glauca.

3 to 4 feet

12.50

$$
4 \text { to } 5 \text { feet }
$$

15.00

Tripetala. 4 to 5 feet.......................................... 10.00

MORUS-Tartarica Pendula. Tea's Weeping Mulberry-

$$
2 \text { year head. }
$$

$12.50 \quad 110.00$

PLATANUS-Orientalis. European Sycamore Plane-

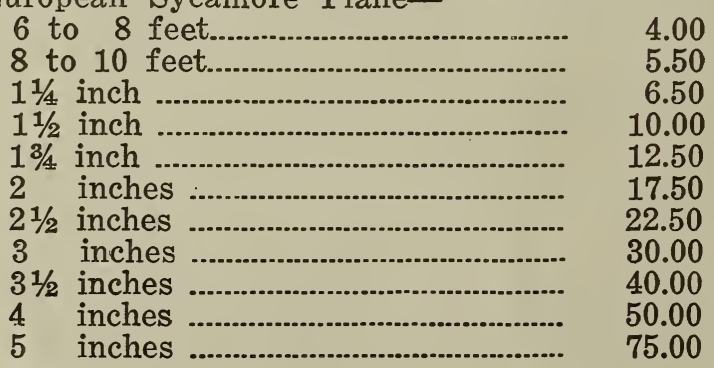

POPULUS-Alba Bolleana. Silver Poplar-

8 to 10 feet.

$11 / 2$ inch 
W. B. COLE, NURSERYMAN, PAINESVILLE, OHIO

POPULUS-Aurea. Golden-leaved Poplar. 8 to 10 feet.

Per 10 Per 100

Monolifera. Carolina Poplar.

$\$ 4.00 \$ 35.00$

8 to 10 feet

3.50

30.00

10 to 12 feet.

4.50

2 inches

12.50

Nigra Pyramidalis. Lombardy Poplar-

$$
\begin{aligned}
& 8 \text { to } 10 \text { feet } \\
& 10 \text { to } 12 \text { feet. } \\
& 11 / 2 \text { inch } \\
& 2 \text { inches .......... }
\end{aligned}
$$

QUERCUS-Fastigiata. Pryamidal Oak.

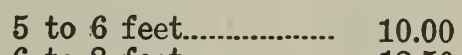

Palustris. Pin Oak.

Rubra. Red Oak.

5 to 6 feet

12.50

6 to 8 feet.

8.00

11.00

75.00

$1 \frac{1 / 2}{\text { inch }}$

17.50

100.00

$13 / 4$ inch

20.00

2 inches

25.00

$21 / 2$ inches

30.00

3 inches

40.00

5 to 6 feet.

8.00

6 to 8 feet

11.00

75.00

$11 / 2$ inch

$13 / 4$ inch

17.50

2 inches

20.00

$21 / 2$ inches

25.00

30.00

SALIX - Dolorosa. Wisconsin Weeping Willow-

8 to 10 feet

$11 / 2$ inch

7.50

Pentandra Laurifolia. Laurel-leaved Willow-

8 to 10 feet.

Vitellina Aurea. Golden-barked Willow-

8 to 10 feet.

10 to 12 feet.

SALISBURIA-Adiantifolia. Ginkgo Biloba, Maiden Hair Tree

$$
6 \text { to } 8 \text { feet... }
$$

2 inch

SORBUS-Aucuparia. European Mountain Ash-

Quercifolia. Oak-leaved Mountain Ash. 6 to 8 feet

TILIA-Americana. American Linden.

Europea. European Linden.

$\begin{array}{lr}6 \text { to } 8 \text { feet....................... } & 5.00 \\ 8 \text { to } 10 \text { feet........................ } & 7.50 \\ 11 / 2 \text { inch ............................. } & 12.50 \\ 13 / 4 \text { inch .......................... } & 15.00 \\ 2 \text { inches ......................... } & 17.50 \\ 21 / 2 \text { inches ......................... } & 25.00\end{array}$


W. B. COLE, NURSERYMAN, PAINESVILLE, OHIO

ULMUS-Americana. White or American Elm-

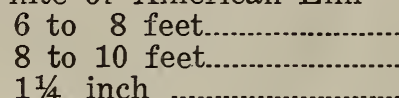

Per 10

$\$ 4.50$

5.50

8.00

$11 / 4$ inch

$11 / 2$ inch

11.00

2 inches

19.00

$2 \frac{1}{2}$ inches

3 inches

$31 / 2$ inches

25.00

30.00

40.00

Campestris. English Cork Elm.

8 to 10 feet.

8.00

$1 \frac{1 / 2}{}$ inch

12.50

Latifolia. Scotch Elm.

6 to 8 feet

4.50

8 to 10 feet

5.50

$11 / 4$ inch

8.50

$11 / 2$ inch

11.00

$13 / 4$ inch

13.50

2 inches

19.00

$2 \frac{1}{2}$ inches

25.00

3 inches

30.00

Montana Pendula.-_Camperdown Weeping Elm-

3 year head.

15.00

We reserve the privilege of making an additional charge for trees of special grade or quality, personally selected by purchaser.

\section{Evergreens}

Prices include balling and burlapping roots.

ABIES-Balsamea. Balsam Fir.

Per 10

$\$ 8.50$

Per 100

2 feet

12.50

3 feet

15.00

5 feet

20.00

Douglasi. Douglas' Spruce.

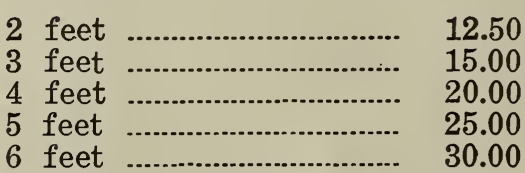

Nordmanniana. Nordman's Silver Fir.

$1 \frac{1}{2}$ feet

12.50

2 feet

15.00

15.00

Veitchii. Japanese Fir. $1 \frac{1 / 2}{2}$ feet

25.00

30.00

17.50

22.50

Nutkaensis Glauca Pendula. 21/2 feet.

17.50

10.00

Chinensis Aurea. Golden. 15 inches

Chinensis Procumbens. Pendulous.

40.00

50.00

75.00

100.00

125.00

175.00

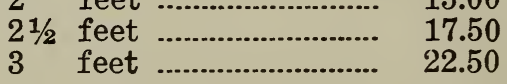


W. B. COLE, NURSERYMAN, PAINESVILLE, OHIO

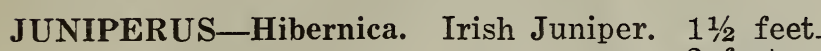

2 feet.

Per 10

$\$ 5.00$

3 feet

6.50

4 feet

8.50

12.50

Japonica Aurea. Japanese Golden Juniper-

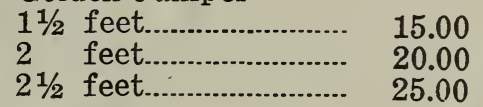

Sabina. Savin Juniper, spreading-

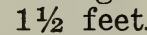

12.50

Sabina Tameriscifolia. Trailing Juniper-

15 inches

12.50

18 inches

15.00

Virginiana. Red Cedar. 2 feet............................. 12.50

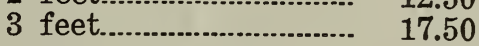

Virginiana Glauca. Blue Cedar. 2 feet.......... 15.00

$2 \frac{1}{2}$ feet........... 20.00

Virginiana Schotti. Golden Cedar. 3 feet.......... 22.50

PICEA_Alba. White Spruce. 2 feet............................................. 8.50

3 feet.............................................. 11.50

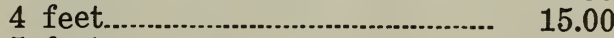

5 feet............................................... 20.00

Engelmanii Glauca. Blue. 2 feet................................... 30.00

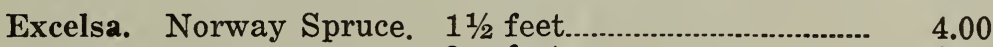

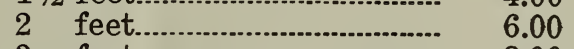

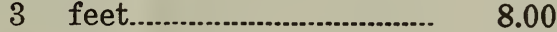

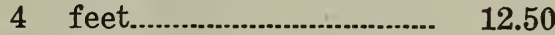

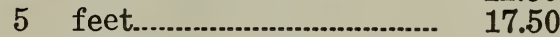

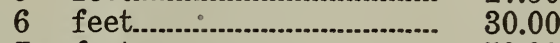

7 feet...................................... 50.00

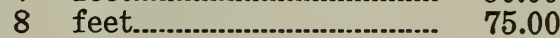

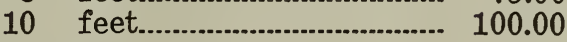

Excelsa Pygmea. Dwarf Norway Spruce-

15 inches............................... 17.50

Excelsa Remonti. Globe shaped Spruce-

$11 / 2$ feet......................... 20.00

Excelsa Pyramidalis. Pyramidal Spruce-

4 feet............................ 30.00

Pungens. Colorado Green Spruce.. 3 feet....................... 15.00

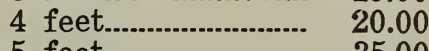

5 feet....................... $\quad 35.00$

Pungens Glauca. Colorado Blue Spruce-

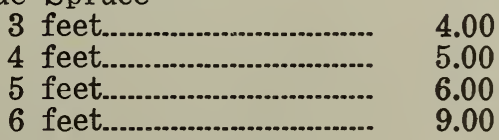

Pungens Glauca Kosterii. Koster's Blue Spruce-

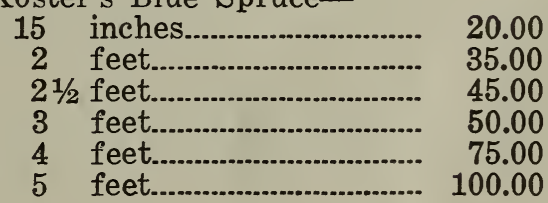




\section{W. B. COLE, NURSERYMAN, PAINESVILLE, OHIO}

PINUS-Austriaca. Austrian Pine.

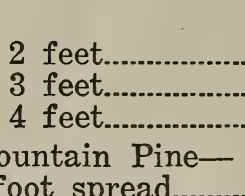

Per 10

$\$ 17.50$

25.00

35.00

Montana Mugho. Dwarf Mountain Pin

$\begin{array}{ll}11 / 2 \text { foot spread............................... } & 15.00 \\ 2 & \text { feet spread......................... }\end{array}$

Strobus. White Pine. 2 feet

10.00

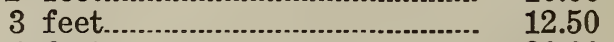

4 feet........................................ 20.00

5 feet............................................ 25.00

6 feet.............................................. 30.00

7 feet.............................................. 60.00

8 feet................................................ 80.00

10 feet................................................. 100.00

Sylvestris. Scotch Pine. 4 feet....................................... 20.00

5 feet......................................... 25.00

6 feet..................................... 30.00

7 feet

8 feet............................................. 80.00

RETINOSPORA-Filifera. Thread-branches Japan Cypress-

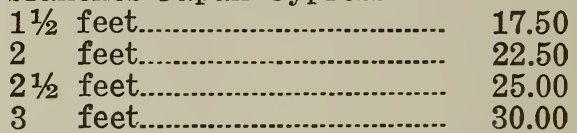

Filifera Aurea. Golden thread-branched Japan Cypress. 15 inches........

Obtusa Nana. Dwarf Cypress. 12 inches..... 12.50

Pisifera. Pea-fruited Japan Cypress-

$\begin{array}{ll}3 \text { feet........... } & 17.50 \\ 4 \text { feet..........- } & 22.50 \\ 5 \text { feet........... } & 27.50\end{array}$

Pisifera Aurea. Golden pea-fruited Japan

Per 100

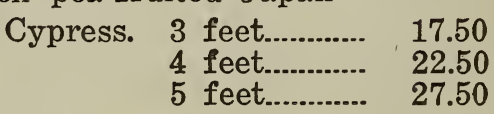

Plumosa. Plume-like Cypress-

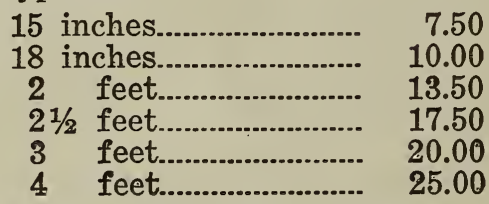

Plumosa Aurea. Golden plume-like Cypress-

15 inches........................ 7.50

18 inches......................... 10.00

2 feet......... 13.50

$21 / 2$ feet......................... 17.50

3 feet..................... 20.00

4 feet............................. 25.00

Plumosa Argentia. Silver Variegated

Cypress. 2 feet................ 15.00

$21 / 2$ feet..................... 20.00

3 feet................... 25.00

Plumosa Compacta Aurea.

Dwarf golden plume-like Cypress- 
W. B. COLE, NURSERYMAN, PAINESVILLE, OHIO

RETINOSPORA-Squarrosa Veitchii. Silver Cypress-

18 inches

2 feet

3 feet.

4 feet.

Per 10

$\$ 12.50$

15.00

20.00

25.00

THUYA-Occidentalis. American Arbor Vitae-

18 inches

2 feet...............................

feet

4 feet

5 feet.

6 feet

Occidentalis Columbia

Silver

feet

3.00

5.00

6.50

9.00

13.50

17.50

20.00

15.00

20.00

9.00

12.50

11.00

13.50

16.00

20.00

25.00

Occidentalis Hoveyii. Hovey's Arbor Vitae-

$$
15 \text { inches }
$$

2 feet

Occidentalis Lutea. (Geo. Peabody) Golden Arbor Vitae.

$$
\begin{array}{ll}
2 & \text { feet.. } \\
21 / 2 & \text { feet.. } \\
3 & \text { feet... }
\end{array}
$$

Occidentalis Pyramidalis. Pyramidal Arbor Vitae-

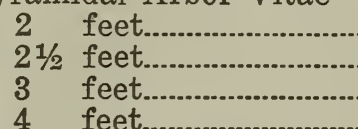

Occidentalis Warreana. Siberian Arbor Vitae-

$$
11 / 2 \text { feet. }
$$

2 feet

Occidentalis Warreana Lutescens. Golden Siberian2 feet. $21 / 2$ feet.

Orientalis. Oriental Arbor Vitae. 2 feet

Orientalis Aurea. Chinese Golden Arbor Vitae-

$$
18 \text { inches. }
$$

TAXUS-Baccata Aurea. Golden Yew. 12 inches.

Cuspidata Brevifolia. Japanese Yew. 15 inches.

Fastigiata Aurea. Golden Irish Yew. 3 feet.

$$
2 \text { feet......................... } 15.00
$$

$21 / 2$ feet........................... 17.50

3 feet............................ 20.00

4 feet........................ 27.50

5 feet............................ 35.00 


\section{Deciduous Shrubs}

ALTHEA-(See Hibiscus)

Per 10 Per 100

AMELANCHIER-Botryapium. Dwarf Juneberry. 2 feet........ $\$ 2.00$

AMORPHA FRUITICOSA. False Indigo. 2 to 3 feet................ 2.00

ARALIA-Pentaphylla. 2 to 3 feet.

1.80

$\$ 18.00$

AZALEA-Mollis 15 inches.

12.50

Pontica. (Ghent) 18 inches.

15.00

Nudiflora. Pink flowering. 18 inches

7.50

2 feet.

10.00

BERBERRIS-Thunbergii. Japanese Barberry 12 inches

1.00

15 inches..................... 1.25

18 inches.

6.00

9.00

1.50

11.00

BUDDLEIA-Summer Lilac (Butterfly Bush).

1.80

CALYCANTHUS-Floridus. Strawberry Shrubs. $1 \frac{11 / 2}{2}$ to 2 feet

1.80

2 to $2 \frac{1}{2}$ feet

Precox. 18 inches

2.00

15.00

1.50

CARAGANA-Arborescens. 2 to 3 feet.

3 to 4 feet

1.60

2.00

4 to 5 feet

2.50

CERCIS-Canadensis. Judas Tree. (Red Bud). 2 to 3 feet....

2.50

3 to 4 feet....

4 to 5 feet....

3.00

20.00

4.00

25.00

35.00

CHIONANTHUS-Virginica. White Fringe. 2 to 3 feet...

5.00

ClETHRA-Alnifolia. Sweet Pepper Bush. $1 \frac{112}{2}$ to 2 feet........

2.00

2.50

2.00

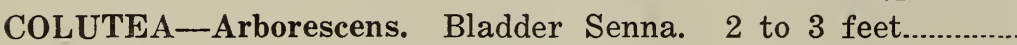

CYTISUS-Laburnum. Golden Chain. 4 to 5 feet

3.00

CORNUS-Florida. White Flowering Dogwood. 2 to 3 feet......

3 to 4 feet.......

4 to 5 feet......

5 to 6 feet.......

Florida Rubra. Red Flowering Dogwood 2 to $2 \frac{1}{2} \mathrm{ft}$.

Alba Elegantissima. Silver-leaved Dogwood. 2 to $3 \mathrm{ft}$.

3 to $4 \mathrm{ft}$.

Alba Siberica. Red-Stemmed Dogwood. 2 to 3 feet....

3 to 4 feet.....

4 to 5 feet....

Alba Spathii. Golden-leaved Dogwood. 2 to 3 feet.... 3 to 4 feet....

2.50

4.00

5.00

7.50

6.00

18.00

22.00

18.00

2.50

3.00

22.00

35.00

1.50

1.80

3.00

2.50

3.00

Stolonifera. Red Osier Dogwood. 2 to 3 feet.......... 1.50

2.00

55.00

22.00

25.00

12.00

15.00

25.00

22.00

25.00

3 to 4 feet.

18.00

Stolonifera Lutea. Yellow-Stemmed Dogwood-

$$
1 \frac{1 / 2}{2} \text { to } 2 \text { feet. }
$$

Oxycantha. Double White Hawthorn. 3 to 4 feet

Oxycantha Paulii. Paul's Double Scarlet

$$
\text { Hawthorn. } 3 \text { to } 4 \text { feet. }
$$


W. B. COLE, NURSERYMAN, PAINESVILLE, OHIO

HYDRANGEA-Arborescens Grandiflora. Early flowering- Per 10 $\begin{array}{ll}11 / 2 & \text { to } 2 \text { feet............. } \\ 2 & \text { to } 3 \\ 3 & \text { to } 4 \text { feet............... }\end{array}$

Paniculata (type) Late flowering. $1 \frac{1 / 2}{2}$ to 2 feet

$\$ 2.20$

2.50

3.50

2 to 3 feet

Paniculata Grandiflora. $1 \frac{112}{2}$ to 2 feet.

$1.70 \quad 15.00$

$2.50 \quad 20.00$

$1.70 \quad 15.00$

$2.20 \quad 20.00$

HYPERICUM-Aureum. Double yellow.

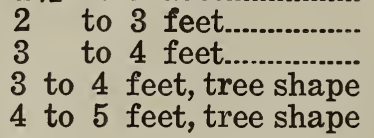
3.00

4.50

5.50

Moserianum. Gold flower.

2.50

2.00

HIPPOPHEA RHAMNOIDES-Sea Buckthorn. 3 to 4 feet......

3.00

ILEX-Verticillata. Scarlet fruit. $1 \frac{1 / 2}{2}$ to 2 feet

2.50

2 to 3 feet.

3.00

3 to 4 feet.

4.00

KERRIA-Japonica. Single Globe Flower. 2 to 3 feet.............. $\quad 2.00$ Japonica flore pleno. Double Globe Flower. 2 to $3 \mathrm{ft}$. $\quad 2.50$

Japonica Variegata. Silver variegated-leaved Corchorus. $1 \frac{1 / 2}{2}$ to 2 feet. 2 to $2 \frac{1}{2}$ feet.............

KOELREUTERIA-Paniculata. Varnish Tree. 4 to 5 feet....... 5 to 6 feet....... 6 to 8 feet...

Per $\mathbf{1 0 0}$ Per $\mathbf{1 0 0 0}$

LIGUSTRUM-Amurense. Amoor River Privet. $1 \frac{112}{2}$ to 2 feet $\$ 8.00$ 2 to 3 feet

10.00

8.00

Ibota. Chinese Privet. $1 \frac{11}{2}$ to 2 feet

10.00

Ibota Regelianum. Regel's Privet. 1/1/2 to 2 feet 15.00

20.00

Ovalifolium. California Privet. 1 to $11 / 2$ feet $11 / 2$ to 2 feet 2 to 3 feet

Vulgaris. English Privet. 1 to $1 \frac{1 / 2}{2}$ feet......... $1 \frac{1}{2}$ to 2 feet.......... 2 to 3 feet.

2.00

2.80

3.50

$\$ 70.00$ 90.00 70.00 90.00

3.50

5.00

6.50

18.00

25.00

30.00

30.00

45.00

Per 10 Per 100

LINDERA-Benzoin. Spice Bush, 11/2 feet. $\$ 2.00$

LONICERA-Upright Honeysuckle-

Bella Albida, 2 to 3 feet

Chrysantha. 3 to 4 feet.

Fragrantissima. Fragrant, small white flowers-

Ledebouri. 3 feet 2 to 3 feet.

Morrow. 2 to 3 feet

Tartarica Alba. 2 to 3 feet

Tartarica Rosea. 2 to 3 feet. 
MALUS-Ioensis. Bechtel's double flowering Crab. 2 to 3 feet

Parkmanii. 3 to 4 feet.

3 to 4 feet

$$
4 \text { to } 5 \text { feet. }
$$

Per 10

$\$ 4.50$

5.50

4.50

6.00

OXYDENDRON-Arboreum. (Andromeda) Sorrel Tree-

$$
1 \frac{1 / 2}{2} \text { to } 2 \text { feet. }
$$

PHILADELPHUS-Syringa or Mock Orange-

Unnamed Sorts. 3 to 4 feet.

4 to 5 feet.

5 to 6 feet.

1.70

2.20

3.00

Coronarius. Sweet scented Syringa-

$$
\begin{aligned}
& 3 \text { to } 4 \text { feet } \\
& 4 \text { to } 5 \text { feet. }
\end{aligned}
$$

Coronarius Aureus. Golden leaved-

$$
1 \text { to } 1 \frac{1 / 2}{1} \text { feet. }
$$

Gordonianus.

$$
2 \text { to } 3 \text { feet. }
$$

3 to 4 feet

4 to 5 feet.

5 to 6 feet.

Grandiflorus.

$$
3 \text { to } 4 \text { feet. }
$$

5 to 6 feet

Lemoinei. Dwarf. $1 \frac{1 / 2}{2}$ to 2 feet

$$
2 \text { to } 3 \text { feet.................... }
$$

Lemoinei Avalanche. Very dwarf. 2 to $2 \frac{1}{2} \mathrm{ft}$.

Lewisii. 2 to 3 feet.

3 to 4 feet

4 to 5 feet.

5 to 6 feet.

Mont Blanc. 2 to 3 feet

$$
3 \text { to } 4 \text { feet. }
$$

PRUNUS-Japonica Alba fl. pl. Double White-flowering Almond

$$
2 \text { to } 3 \text { feet }
$$

Japonica Rosea fl. pl. Double Pink flowering Almond-

$$
2 \text { to } 3 \text { feet. }
$$$$
3 \text { to } 4 \text { feet }
$$

Pissardi. Purple-leaved Plum. 3 to 4 feet.

PYRUS-Arbutifolia. Red Chokeberry. $1 \frac{1}{2}$ to 2 feet.

Cotinus. Purple Fringe or Smoke Tree. 3 to 4 feet......

\subsection{0}

18.00

22.00

25.00 
RHUS-Glabra. Smooth Sumach. 4 to 5 feet.

Per 10

Per 100

Glabra Laciniata. Cut-leaved Sumach.

2 to 3 feet......

$\$ 2.50$

$\$ 22.00$

Typhina. Staghorn Sumach. 3 to 4 feet.

4.00

3 to 4 feet

5.00

Typhina Laciniata. Cut-leaved. 3 to 4 feet.

2.50

3.00

4 to 5 feet.

4.00

RIBES-Aureum. Yellow Flowering Currant. 2 to 3 feet.

1.70

3 to 4 feet........ $\quad 2.20$

15.00

4 to 5 feet.

3.00

20.00

Sanguineum. Red flowering Currant. 2 to 3 feet......

2.20

27.00

3 to 4 feet....

RUBRES ODORATUS. 2 to 3 feet.

3.00

2.00

3 to 4 feet.

2.50

SAMBUCUS-Canadensis. Common Elder. 3 to 4 feet.

1.70

20.00

27.00

Canadensis Acutiloba. Cut-leaved Elder-

4 to 5 feet.

2.20

15.00

3 to 4 feet.

2.00

20.00

4 to 5 feet

3.00

18.00

Nigra Aurea. Golden-leaved Ender. 2 to 3 feet

1.70

3 to 4 feet

2.20

27.00

4 to 5 feet

15.00

20.00

$3.00 \quad 27.00$

SPIREA-Anthony Waterer. Dwarf pink flowering-

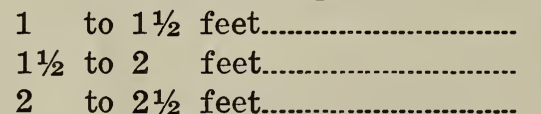

1.70

15.00

2.20

20.00

Arguta Multiflora. White flowering. 3 to 4 feet......

3.00

2.20

Billardi. Pink flowering. 2 to 3 feet.

1.70

15.00

3 to 4 feet.

2.20

20.00

Callosa Alba. Dwarf white flowering. 1 to $1 \frac{1}{2}$ feet

2.00

Callosa Superba. Dwarf light pink. 1 to $1 \frac{1 / 2}{2}$ feet....

1.70

15.00

Lindleyana. Large compound leaves. 3 to 4 feet......

2.00

18.00

Opulifolia. Cream colored flowers. 3 to 4 feet.........

2.00

18.00

Opulifolia Aurea. Golden leaved. 2 to 3 feet

1.70

15.00

3 to 4 feet.

2.20

20.00

4 to 5 feet.

3.00

5 to 6 feet.

27.00

5.00

Paniculata Rosea. Pink panicles. 3 to 4 feet..............

2.00

18.00

Prunifolia. Bridalwreath. 2 to 3 feet

2.50

Reevesiana. Single white flowering. 2 to 3 feet.........

1.70

15.00

3 to 4 feet.........

2.20

20.00

Reevesiana fl. pl. Double white flowering. 2 to $3 \mathrm{ft}$.

Salicifolia. Willow-leaved Spirea. 2 to 3 feet.

2.00

18.00

Thunbergii. Early single white flowering. 2 to 3 feet

2.00

18.00

3 to 4 feet

3.00

Tomentosa. Pink cone-shaped flowers. 2 to 3 feet....

1.70

15.00

3 to 4 feet....

2.20

20.00

Van Houttei. Single white flowering. 2 to 3 feet.... 


W. B. COLE, NURSERYMAN, PAINESVILLE, OHIO

SOPHORA-Japonica. Japan Pagoda Tree. 3 to 4 feet.. STEPHENANDRA-Flexuosa. Creamy white flowers 2 to 3 feet STYRAX-Japonica. 2 to 3 feet...

SYMPHORICARPUS-Racemosus. Snowberry or Waxberry2 to 3 feet. 3 to 4 feet.

Vulgaris. Coralberry or Indian Currant-

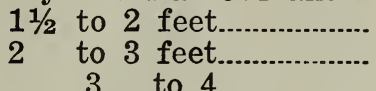

SYRINGA-Japonica. Japanese tree lilac. 2 to 3 feet............... Josikea. Hungarian Lilac. 3 to 4 feet... Persica. Persian Lilac, Pink. 2 to 3 feet.. 3 to 4 feet.

Persica Alba. Persian Lilac, White. 2 to 3 feet.... Rothomagensis. Red flowering. 2 to 3 feet........ Villosa. Pale Lilac. 2 to 3 feet... Vulgaris. Common purple Lilac. 2 to 3 feet........ 3 to 4 feet.........

Vulgaris Alba. Common white Lilac. 2 to 3 feet 4 to 5 feet

Vulgaris. Following named sorts. 2 to 3 feet...... 3 to 4 feet......

Alphonse Lavalle. Double blue.

Belle de Nancy. Double rose.

Charles X. Single reddish purple.

LaTour d'Avergne. Double red.

Ludwig Spaeth. Single red.

Madame Abel Chateney. Double white.

Madame Casimir Perier. Double creamy. white.

Madame Lemoine. Double white.

Marie LeGraye. Creamy white.

President Grevy. Double white.

TAMARIX - Africana. Early, light pink. 3 to 4 feet

$$
4 \text { to } 5 \text { feet. }
$$

Hispida Aestivalis. Late pink. 3 to 4 feet...

Odessana. Bluish green foliage. 3 to 4 feet.

VIBURNUM-Dentatum. Dentated leaves, blue berries2 to 3 feet.

Lantana. White flowering. 2 to 3 feet.

3 to 4 feet.

4 to 5 feet

Opulis. High bush Cranberry. Scarlet berries-

$$
2 \text { to } 3 \text { feet.. }
$$

3 to 4 feet.

4 to 5 feet.

Opulis Sterile. Common Snowball. 2 to 3 feet.. 3 to 4 feet..

4 to 5 feet..

Opulis Nana. Very dwarf. 10 to 12 inches........

Tomentosum Plicatum. Japan Snowball. 2 to $3 \mathrm{ft}$. 3 to $4 \mathrm{ft}$.
Per 10 $\$ 5.00$

2.00

2.00

2.00

2.50

1.20

1.50

2.00

3.00

2.50

2.50

3.00

2.50

2.50

2.50

1.70

2.20

2.50

5.00

3.00

4.00

Per 100

18.00

18.00

22.00

10.00

13.00

18.00

22.00

22.00

15.00

20.00

2.00

18.00

2.50

22.00

2.50

22.00

2.50

2.00

2.00

2.50

18.00

18.00

22.00

$3.50 \quad 30.00$

$1.70 \quad 15.00$

2.20

20.00

$3.50 \quad 30.00$

$1.70 \quad 15.00$

$2.20 \quad 20.00$

3.50

2.00

18.00

3.00

4.00 


\section{W. B. COLE, NURSERYMAN, PAINESVILLE, OHIO}

WEIGELIA-Mixed sorts. 3 to 4 feet.

Per 10

Abel Carriere. Dark rose.

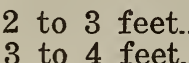

Amabilis. Light pink. 3 to 4 feet.

Candida. White flowering. 3 to 4 feet

Eva Rathke. Crimson flowering. $1 \frac{1 / 2}{2}$ to 2 feet..

2 to 3 feet..

3 to 4 feet..

Lutea. Dwarf; small yellow flowers.

$$
1 \frac{1}{2} \text { to } 2 \text { feet }
$$

Rosea._-Pink flowering. 2 to 3 feet

Rosea Nana Variegata. Variegated-leaved.

$$
11 / 2 \text { to } 2 \text { feet... }
$$$$
2 \text { to } 3 \text { feet }
$$

2.00

2.50

2.00

2.50

2.00

2.50

3.50

2.00

2.00

2.00

2.50

Per 100

$\$ 18.00$

18.00

22.00

18.00

18.00

22.00

30.00

18.00

18.00

22.00

\section{Evergreen Shrubs}

EUONYMUS-Radicans. Green leaved trailing vine.

8 to 12 inches

Per 10

$\$ 1.50$

2.00

Radicans Carrieri. 12 to 15 inches

1.50

Vegetus. Shrubby evergreen Euonymus. 10 to 12 inches.

KALMIA-Angustifolia. Deep rose flowering.

2 to $2 \frac{1}{2}$ feet

Latifolia. Mountain Laurel, 18 inches

12.50

12.50

MAHONIA-Aquifolia. Yellow flowering, holly-leaved.

1 to $1 \frac{1 / 2}{\text { feet. }}$

$1 \frac{1 / 2}{2}$ to 2 feet

2.00

3.00

4.00

1.20

Per 100

PACHYSANDRA-Terminalis. Evergreen ground cover.

LONICERA-Pileata. $\begin{array}{r}4 \text { to } 6 \text { inches................. } \\ \text { Evergreen Honeysuckle. }\end{array}$

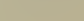

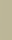

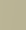

15 to 18 inches.

2.50

20.00

25.00

30.00

\section{Vines}

AMPELOPSIS-Quinquefolia. Virginia Creeper. 2 years........ Quinquefolia Engelmanni. 2 years.....................

Veitchii. Boston Ivy. 2 years..............................

ARISTOLOCHIA-Sipho. Dutchman's Pipe ................................

Per 100

$\$ 13.00$

25.00 
W. B. COLE, NURSERYMAN, PAINESVILLE, OHIO

LONICERA-Aurea Reticulata. Golden variegated-leaved Honeysuckle

Per 10

Per 100

Belgica. Monthly fragrant Honeysuckle.

$\$ 2.00$

2.00

Halleana. Hall's Japan Honeysuckle.

1.50

Sempervirens. Scarlet Trumpet Honeysuckle........ $\quad 2.00$

13.00

LYCIUM-Chinensis. Matrimony Vine

2.00

TECOMA-Grandiflora. Chinese Trumpet Vine..

2.50

Radicans. (Bignonia). Trumpet Vine.

1.50

1.00

8.00

VINCA-Minor. Periwinkle or Myrtle.

2.00

WISTARIA-Magnifica. Blue

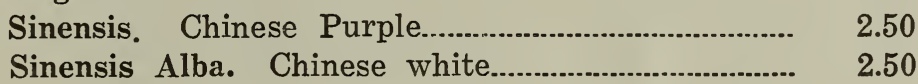

\section{Roses}

HYBRID PERPETUAL-Following varieties, 2 years.

Per 10 Per 100

Anna D. Diesbach. Pink.

Baroness Rothschild. Light pink.

Clio. Blush white.

Coquette des Alpes. White.

Captain Hayward. Red.

Earl of Dufferine. Velvety crimson.

Eugene Furst. Deep, vivid crimson.

Fisher Holmes. Rich Crimson.

Frau Karl Druschki. Pure white.

General Jacquiminot. Brilliant crimson.

George Dickson. Red.
Heinrich Munch. Pink.

Hugh Dickson. Deep red.

J. B. Clark. Red.

Magna Charta. Bright rose.

Madame Gabriel Luzet. Silvery pink.

Margaret Dickson. White.

Marshäll P. Wilder. Bright red.

Mrs. John Laing. Soft pink.

Paul Neyron. Large pink.

Tom Wood. Full cherry red.

Ulrich Brunner. Cherry red.

HYBRID TEA-British Queen. White.

Gruss an Teplitz. Brilliant crimson................... 4.50

$\$ 38.00$

Killarney Pink

4.50

Killarney White

4.50

Madame Caroline Testout. Pink

4.50

Rhea Reid. Dark crimson.

5.00

HYBRID PERNETIANA-Persian Yellow

4.50

Soliel d'Or

5.00

40.00

Willowmere. Salmon pink shaded yellow.

HYBRID SWEET BRIAR-Amy Robsart. Bright rose

Jeanie Deans. Fiery crimson...........

Lord Penzance. Ecru shaded to lemon yellow....

Meg Merrilies. Gorgeous crimson..

HYBRID RUGOSA-Blane de Coubert. Pure white

Belle Poitevine. Double rose 


\begin{tabular}{lllll}
\hline \hline W. B. COLE, NURSERYMAN, & PAINESVILLE, & OHIO \\
\hline \hline
\end{tabular}

RUGOSA-Alba. Single white.

Per 10

John Cranston. Double red.

POLYANTHA-(Dwarf) Baby Rambler. Dark pink

CLIMBING-American Beauty. Large pink.

Baltimore Belle. Blush white

Prairie Queen. Red..

Setigera. Prairie rose. Single pink.

RAMBLER-American Pillar. Large single rosy pink..

Doctor Van Fleet. Double flesh pink.

Flower of Fairfield. Everblooming crimson rambler

Hiawatha. Brilliant scarlet.

Tausendschon. Large clustered pink............................

Veilchenblau. Blue Rambler.

White Rambler

Yellow Rambler .................................................-...............

WICHURAIANA-Memorial Rose ...............................................

Dorothy Perkins. Pink

Dorothy Penkins. White.

Excelsa. Crimson Dorothy Perkins..................

Silver Moon

ROSA BLANDA-Meadow Rose

ROSA NITIDA-Shining leaf rose.

\section{Perennial Plants}

ACHILlEA-The Pearl. White, June to August.

Per 10

$\$ .70$

.80

.90

Flos Jovis. Pink, June.

ALTHEA-Rosa (Hollyhock). Separate colors, double and single ALYSSUM-Saxatile Compactum. Yellow, May..

ANEMOME-Queen Charlotte. Rose, fall

Rubra. Red, fall.

Whirlwind. Pure white, fall

ANCHUSA-Italica. Blue, June to September.

ANTHEMIS-Tinctoria. Yellow, July.

AQUILEGIA-Canadensis. Scarlet, May and June.

Chrysantha. Yellow, June.

Coerulea Hybrids. Various, June.

Nivea Grandiflora. White, June

Skinneri. Red and yellow, May.

ASTER-Nova Anglae. Purple, September and October.

Snow Queen. White, September and October.

St. Egwyn. Soft pink, September and October...

BAPTISIA-Australis. Indigo, June.

BOCCONIA-Cordata. Creamy white, August.

BOLTONIA-Asteroides. White, September..

Latisquama. Pink, August.

.90

.80

1.00

1.00

1.00

1.20
3.50

3.00

4.50

4.50

4.50

Per 100 30.00 25.00 40.00 40.00 40.00

45.00

4.00 。 $\quad 35.00$

$3.00 \quad 25.00$

$3.00 \quad 25.00$

$2.50 \quad 20.00$

$4.00 \quad 35.00$

$4.00 \quad 35.00$

4.50

4.00

3.50

4.00

3.50

3.00

3.50

2.00

3.50

3.00

3.00

4.50

1.80

1.80

15.00

15.00

30.00

25.00

30.00

15.00

30.00

25.00

25.00

Per 100

$\$ 6.00$

7.00

8.00

8.00

7.00

9.00

9.00

9.00

10.00 


\begin{tabular}{lllll}
\hline W. & B. & COLE, NURSERYMAN, & PAINESVILLE, OHIO \\
\hline \hline
\end{tabular}

CAMPANULA-Carpatica. Blue, July to September

Per 10

$\$ .80$

Carpatica Alba. White, July to September......

Media. Separate colors, June and July.

Media Calycanthema. Separate colors

Persicifolia. Blue, June to July.

Pyramidalis. Blue, July

CASSIA-Marylandica. Yellow, July.

CENTAUREA-Montana. Blue

CERASTIUM-Tomentosum. White

CHRYSANTHEMUM-October. Following hardyvarieties

Ashbury. Sulphur white.

Autumn Glow Red.

Dawn. Pink. Pompon.

Eleanor. White.

Eva. Dwarf, deep pink.

Garza. Large quilled white.

Germania. Large straw white.

Gertrude. White.

James Boone. Pompon. Straw colored.

Nellie Bly. Blush white.

Nellie Rainsford. Light pink.

Rev. W. H. Hoffman. Bronze yellow.

Rosy Morn. Silver pink.

Ruby Queen. Dark garmet. Pompon.

Sylvia. Scarlet bronze.

White Flora. Large white.

Yellow. Large flowered.

CHRYSANTHEMUM-Maximum Shasta (Shasta Daisy. White, July to October.

CONVALLARIA-Majalis. Lily of the Valley. April..................

COREOPSIS-Lanceolata. Golden yellow, summer........................

Tripteris. Yellow, August

.80

1.00

1.00

.80

Chinensis, Blue, June to August.

Hybrids, Gold Medal. Shades of blue

DIANTHUS-Barbatus. Single Sweet William. May and June....

Barbatus fl. pl. Double Sweet William. May and June

Barbatus Newport Pink. Double pink. May and June

Caryophyllus. Hardy Carnation, mixed colors

Latifolius Atrococcineus ff. pl. Double deep crimson ..................................

Plumarius. Scotch Pink. Mixed, June and July

Plumarius Semperflorens. Pink

Plumarius Essex Witch. Old-fashioned hardy garden pink

Plumarius White Reserve

DIGITALIS-(Fox Glove) Rose and white, June and July..........

EULALIA-Gracilima Univitata. Hardy plume grass

Japonica Variegata. White striped hardy plume grass

Japonica Zebrina. Zebra striped hardy plume grass 


\section{W. B. COLE, NURSERYMAN, PAINESVILLE, OHIO}

(a)

FUNKIA-Coerulea. Large flowered blue, July.

Per 10

Per 100

Lanceolata. Plantain Lily. Lilac, July.

$\$ \quad .90$

$\$ 8.00$

Subcordata Grandiflora. White Day Lily. Fragrant

1.50

7.00

Undulata Variegata. White and green foliage.

1.20

12.00

.80

10.00

GAILLARDIA-Grandiflora. Blanket flower. Yellow, crimson

$$
7.00
$$

GYPSOPHILA-Paniculata. Baby's Breath, White, June, July....

HELENIUM-Autumnale. Yellow, August.

HELIANTHUS-(Hardy Sunflower)

Mollis Grandiflorus. Lemon yellow, August..

HELIOPSIS-Pitcheriana. Orange, June to August.

HEMEROCALIS-(Yellow Day Lily)

Flava. Lemon yellow, June.

Minor. Dwarf lemon yellow, first of June....

Thunbergii. Lemon yellow, July.

HESPERIS-Matronalis. Pink, June and July.

HIBICUS-Crimson Eye. White, crimson center. August and September.

Moscheutos. Swamp Rose Mallow. August and

\section{September.}

Mallow Marvels. Pink, August. and September......

Mallow Marvels. White. August and September..

Mallow Marvels. Red. August and September......

Mallow Marvels. Mixed

IRIS-Germanica-Celeste. Azure Blue.

Florentina. Light blue.

\section{Florentina Alba.}

White.

Fulda

Her Majesty. Pink

Honorabilis. Golden yellow, falls mahogany

Madame Chereau. White, fringed blue..

Midnight. Very dark blue.

Mrs. H. Darwin. White, veined crimson.....

Parasensis. Deep blue.

Queen of the May. Soft, rosy lilac.

Gerda. Creamy yellow, falls darker yellow..

Halfdan. Creamy white. 
W. B. COLE, NURSERYMAN, PAINESVILLE, OHIO

IRIS-Kaempferi. No. 16. Deep blue, yellow throat, 6 petaled

Per 10

No. 23. Royal purple, orange throat, 6 petal

Siberica. Purplish blue.

$\$ \quad .90$

Per 100

.90

$\$ 8.00$

.70

8.00

.80

6.00

LATHYRUS-Latifolius. (Perennial Pea) Pink, June, July 7.00

LAVANDULA-Vera. Sweet Lavender. July and August....

LIATRIS-Spicata. Purple, September.

1.00

LILIUM-Canadense. Yellow to red. June to August..

1.50

Candidum. Madonna Lily. White, June.

2.00

LINUM-Perenne. (Flax) Blue flowers, all summer.

Perenne Album. White flowers

LOBELIA-Cardinalis. Scarlet, August and September.

1.00

LYCHNIS-Chalcedonica. (Maltese Cross) Scarlet, July.

MONARDA-Didyma. Cambridge Scarlet. August to Sept.......

MYOSOTIS-Palustris. (Forget-me-not) Azure blue, May, June

PEONY_Officinalis Rosea. Rose pink, very early

Officinalis Rubra. Dark red, very early.

2.00

Sinensis. Strong plants, three to five eyes in the

following varieties:-

Andre Laurie. Large deep rose, late............

Alba Nivea Plena. Double white..................

2.00

18.00

Canary. Straw colored

2.00

18.00

Delachi. Deep crimson, late..............................

2.00

18.00

Duchess de Nemours. Large creamy white

2.00

18.00

Duke of Wellington. Sulphury white.

2.00

18.00

Duc de Cazes. Brilliant carmine pink..........

2.00

18.00

Edulis Superba. Bright rose pink, early....

2.00

Felix Crousse. Brilliant crimson...................

5.00

Festiva Alba. White................................... 2.00

Festiva Maxima. Pure white, early............

Fragrans. Pink.

Humeii. Deep rose, late.

2.00

18.00

Jeanne d'Arc. Sulphur white, pink center..

2.00

18.00

LaTulipe. Blush white.

18.00

L'Eclatante. Purplish crimson.

2.50

22.00

2.00

2.00

Louis Van Houtte. Deep crimson..

18.00

Madame Breon. Shell pink.

Marie Lemoine. Creamy white, very late....

Modeste Guerin. Deep rose

Madame Calot. Light pink

Mons. Jules Elie. Peerless pure pink...........

President Roosevelt. Deep red, very late....

Queen Victoria. Blush white. 


\section{W. B. COLE, NURSERYMAN, PAINESVILLE, OHIO}

PAPAVER-Orientale. Oriental Poppy.

Per 10

Nudicaule. White and yellow

PENTSTEMON-Barbatus Torrevi. Coral red, July and August

PHLOX_Paniculata. (Decussata). Following named varieties:

Alceste. Violet shading to light blue........

Athis. Salmon pink.

B. Comte. Brilliant French purple.............

Coquelicot. Orange scarlet..

Eclaireur. Brilliānt rosy magenta, white-eye

Huxley. Pink

Independence. Large, pure white.................

LaVogue. Clear, silvery rose.......................

LeMahdi. Violet blue

Madame Bezanson. Scarlet.............................

Mrs. Dwyer. Pink eye.

Pantheon. Rosy pink.

Perle du Nord. Pure white, distinct red eye

Princess Louise. White, pink eye...............

Rosenberg. Large magenta

Richard Wallace. White, crimson eye......

Sir Edward Landseer. Brilliant crimson....

Sunset. Deep rosy pink

Suffruiticosa. Miss Lingard. Early waxy white...........

Subulata Rosea. Creeping Moss Pink.

PHYSOSTEGA-Virginica. Pink and white mixed, August........

Virginica Rosea. Pink

Virginica Alba. White

PLATYCODON-Grandiflorum. Blue

Grandiflorum Album. White.

Mariesi. Dwarf blue, July to October.

PLUMBAGO-Larpentae. Dwarf blue, September and October

PYRETHRUM-Roseum. Shades of Pink and red mixed, May and June

Uliginosum. White, September

RUDBECKIA_Laciniata (Golden Glow) Double yellow,

August and September.

Purpurea. Purple cone flower.

SALVIA-Azurea Grandiflora. Sky blue, August and September Argentea. Pinkish white, June

Pitcheriana. Blue, August.

SAPONARIA-Ocymoides. Pink, May and August..

SCABIOSA-Caucasica. Lavender, all summer.

SEDUM-Spectabilis. Rose colored, August and September......

Spectabilis Brilliant. Crimson, August and September

STATICE-Latifolia (Sea Lavender) Blue, July and August....

STOKESIA-Cyanea. Blue, August and September

$\$ 1.00$

1.00

.80

.90

.90

1.50

1.20

.90

.90

1.00

.90

1.20

1.00

.80

1.00

.90

.70

.90

.80

.90

.90

.80

.70

.80

.90

.90

.60

.70

.80

1.20

1.00

.90

1.20

1.00

1.00

9.00

8.00 
THERMOPSIS-Caroliniana. Yellow, June and July.

Per 10

TUNICA-Saxifragra. Dwarf compact, light pink, July to Sept.

$\$ 1.00$

VALERIANA-Officinalis. Light pink, June to July.

.80

Per 100

Officinalis Coccinea. Red, June to Octo

1.00

$\$ 9.00$

Maritima. Blue, July to September

1.00

7.00

VERONICA-Maritima. Blue, July to September
Spicata. Blue, June to August...............

9.00

VIOLA--Cornuta, Admiration. Purple, June to September.

Cornuta, Blue Perfection. Deep blue, June to Sept.....

.70

9.00

Cornuta, Lutea Splendens. Yellow, June to September

.90

7.00

6.00

.90

8.00

Cornuta, White Perfection. White, June to September

.90

8.00

Cornuta Mixed. All colors.

.90

8.00

.70

8.00

1.40

6.00

YUCCA-Filamentosa. White, June and July.

12.00




